\title{
Disseminating Emergency Preparedness Planning Models as Automatically Generated Custom Spreadsheets
}

\author{
Jeffrey W. Herrmann \\ Department of Mechanical Engineering and Institute for Systems Research, \\ University of Maryland, College Park, MD 20742 \\ Phone: (301) 405-5433 \\ Fax: (301) 314-9477 \\ Electronic mail: jwh2@umd.edu
}

\begin{abstract}
When public health officials requested operations research models to help county health departments across the United States create plans for dispensing medications and vaccines, we developed capacity planning and queueing network models. We then faced the challenge of distributing these models to a set of persons who have unknown experience with operations research techniques and no resources for acquiring and learning new software. We decided to use spreadsheets, which eliminated the most significant obstacles. To allow users to evaluate a wide variety of plans, we created software that runs macros to generate spreadsheet models based on user input. Public health emergency preparedness planners can download the software from our web site. Developing spreadsheets for this type of application is very different from end-user modeling and typical spreadsheet applications.
\end{abstract}

\section{Introduction}

Montgomery County, Maryland, is home to one of eight Advanced Practice Centers (APCs) for Public Health Preparedness. The APCs customize and package innovative tools for other local public health agencies to strengthen bioterrorism planning and response capabilities. The Centers for Disease Control and Prevention (CDC) funds the APC program. The National Association of County and City Health Officials (NACCHO) administers the program and maintains an online clearinghouse of APC preparedness tools for local public health agencies (NACCHO 2005). The long-term goal is to create and disseminate a broad set of methods and guidelines to help county health departments create, assess, and improve their emergency preparedness and response plans. 
Emergency preparedness includes carefully planning mass dispensing and vaccination clinics, also known as points of dispensing (PODs). The health department must assign the right number of workers to various roles when the clinic begins operations. They must consider the capacity of each clinic (the number of residents it can serve per hour) and the number of minutes residents would spend in the clinic (the time in system, the flow time, or the throughput time). Clinic capacity affects the number of clinics needed and the total time needed to vaccinate the affected population. The time in system affects the number of residents who would be inside the clinic waiting for treatment; too many residents in the clinic can cause crowding and confusion.

Evaluating clinic plans clearly requires the use of operations research models such as capacity planning and queueing. We developed discrete-event simulation models and capacity planning and queueing network models to improve clinic planning in an ongoing collaboration between the University of Maryland, College Park, and the Montgomery County (Maryland) Public Health Services (PHS). Aaby et al. (2006b) provide more information about these models.

In this paper we discuss the challenge of distributing these models to a set of persons who have unknown experience with operations research techniques and no resources for acquiring and learning new software. Providing the models in a useful format was a critical problem. The possible choices included publishing a report that presented the mathematical models, developing an original piece of software, creating spreadsheets for common clinic layouts, and providing discrete-event simulation models. We decided on developing software that creates spreadsheets based on user input.

\section{Emergency Preparedness Planning}

After the September 11, 2001, terrorist attacks, public health officials at the federal, state, and county level accelerated efforts to plan their response to events such as bioterrorism attacks (the intentional release of smallpox or anthrax, for example) and outbreaks of contagious disease (such as pandemic influenza). The resulting plans often call for mass dispensing (or vaccination) clinics. See, for example, 
CDC (2002). Public health officials need to create a set of clinic designs to prepare for a range of emergencies, including smallpox, anthrax, and pandemic influenza.

In the worst-case scenario, if terrorists could release a lethal virus, such as smallpox, into the general population, every person in the affected area would have to be vaccinated within a few days. Kaplan et al. (2002) compare vaccination policies for responding to a smallpox attack and show that mass vaccination results in many fewer deaths than other tactics in the most likely attack scenarios. For example, Montgomery County, Maryland, would need to vaccinate nearly one million people. To vaccinate so many people in a short period it would have to set up PODs at designated sites throughout the county.

At a typical mass vaccination clinic, residents go to the triage station outside the clinic building. Members of the triage station staff ask residents whether they have any symptoms of smallpox (a rash or fever) or know they have been exposed to the smallpox virus. Other staff members escort symptomatic residents to a symptoms room, where they will consult with a doctor. Residents exposed to the virus go to a holding room to wait for medical attention. After consulting with a doctor, infected residents exit the clinic and go to the hospital; healthy residents are allowed continue to registration (Figure 1).

After entering the clinic, residents obtain registration forms and printed information on smallpox at the registration station. (The staff includes translators.) Residents then go to the education station, classrooms in which they watch informational videos about the smallpox vaccine and fill in the registration forms. The education station staff manages the classrooms and checks the registration forms for completeness. Residents then walk to the screening station.

At the screening station, they see medical personnel who check their registration forms and direct residents with possible complications based on their medical histories to visit the consultation station. The remaining residents sign consent forms and go directly to the vaccination station.

At the consultation station, residents discuss possible complications with a doctor. Those who refuse the vaccination receive an information sheet and leave the clinic. They will be monitored by public health officials. Those who decide to be vaccinated sign consent forms and go to the vaccination station. 
At the vaccination station, a vaccination nurse verifies that the consent form has been signed and witnessed and then vaccinates the resident. Another staff member and the resident go over an information sheet about what to do after the vaccination, and then the resident leaves the clinic.

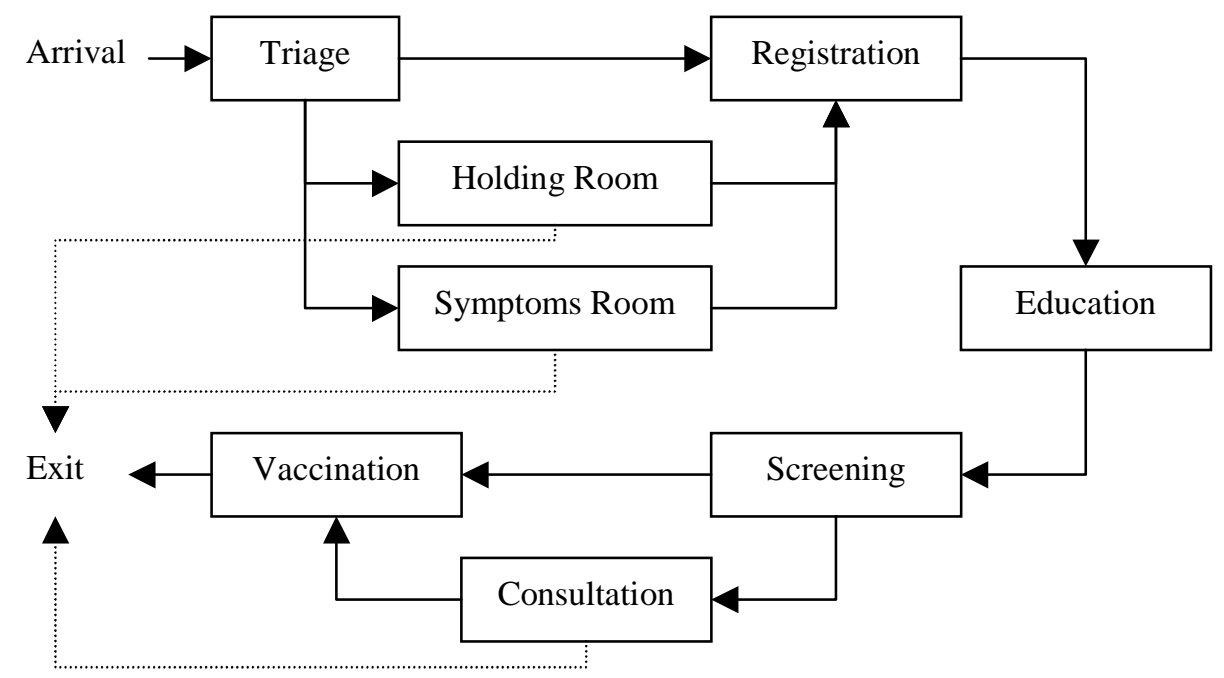

Figure 1: Flowchart of resident flow in a typical mass vaccination clinic (dashed lines show residents who exit without receiving vaccinations).

\section{Choosing Spreadsheets}

We began our analysis of clinic planning with a simulation study to evaluate alternative clinic designs (Aaby et al. 2005). We designed and built a discrete-event simulation model of the massvaccination clinic based on data collected during a full-scale exercise. After validating the model with our results, we used simulation models to evaluate alternative clinic designs and operational policies. Though very useful, discrete-event simulation models require specialized software that public health officials do not have. Thus, we decided to develop capacity planning and queueing network models and implement them as spreadsheets. Other options were considered but discarded, as discussed later.

We first developed two capacity planning and queueing models: one for vaccination against a contagious disease such as smallpox (based on the configuration in Figure 1), and another for dispensing medicine (such as antibiotics in case of anthrax). Public health officials who reviewed these models concluded that these two models did not offer sufficient flexibility to evaluate a wide range of reasonable clinic designs. Therefore, we created software that can generate a spreadsheet-based capacity planning 
and queueing model for a customized clinic configuration. This software is called the Clinic Planning Model Generator. It is available on this web site for public health professionals to download and use: http://www.isr.umd.edu/Labs/CIM/projects/clinic/.

Our software is a helpful tool for creating good plans and improving existing ones. Planners can use the clinic planning models to determine the number of staff members they need to achieve the capacity they need and to design clinics that avoid unnecessary congestion. The models are based on data collected from clinic exercises, and they have been validated by those exercises and by public health professionals.

Public health professionals from around the United States have downloaded the software and used it to help create plans for mass dispensing and vaccination clinics. Aaby et al. (2006a) describes the use of the Clinic Planning Model Generator for planning mass vaccination clinics for pandemic influenza. CDC recommended the model's use in their guidance for pandemic influenza preparation (CDC, 2006).

\section{Software Structure}

The Clinic Planning Model Generator includes software and documentation. The software consists of two Microsoft Excel workbooks: the Clinic Generator and the Clinic Template. The documentation consists of a users guide (a PDF document) and the Sample Clinic Model (a third Microsoft Excel workbook). The code in the workbooks is implemented in Visual Basic for Applications (VBA) macros that run when the workbook is opened or when the user clicks on buttons in the spreadsheet. The generator and template files are 140 kilobytes each, and the sample clinic model is nearly 210 kilobytes. Figure 2 provides a system view of the software.

The Clinic Template contains all of the worksheets and VBA macros that are needed for a planning model. These worksheets contain text, formatted cells, navigation buttons, and many of the equations needed. To create the planning model, VBA macros in the Clinic Generator accept user input, open the template, copy and paste cells from the template as needed, directly write formulas into the worksheets, and save this modified workbook as the planning model. 


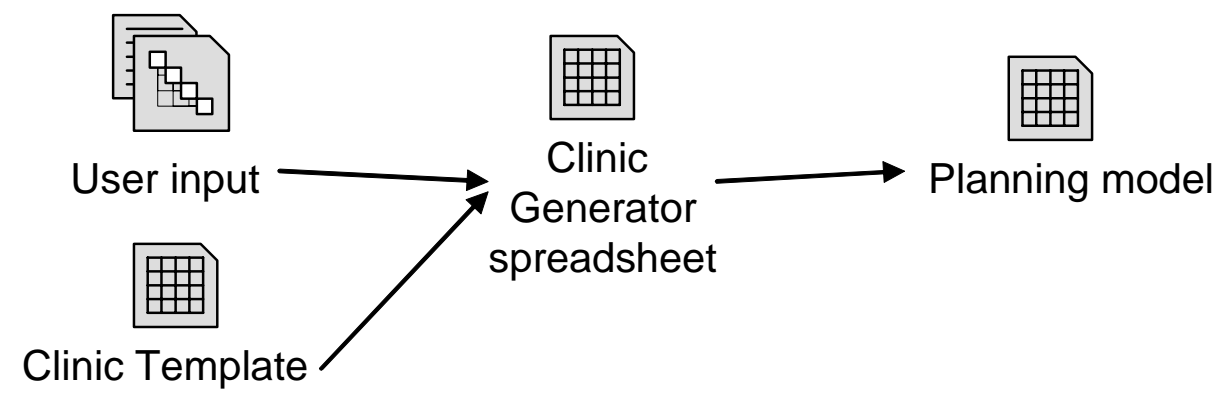

Figure 2: The Clinic Generator software takes inputs from the user and modifies the Clinic Template file to create a custom clinic planning model that the user can modify as desired.

The clinic planning process involves three steps. First, before beginning to build the clinic planning model, the user gathers the information that will be used and determines the flow of residents from one station to another within the clinic. Blank forms are provided in the users guide to help with this task.

Second, the user opens the Clinic Generator workbook, which starts a macro that asks the user for information about the clinic. This macro then modifies the Clinic Template workbook to create and save a new workbook that is the user's clinic planning model.

Third, the user works directly in the new workbook, where they can enter additional information, modify the clinic specifications, and view the performance estimates.

The documentation includes the users guide and the Sample Clinic Model. The users guide defines important terms related to the queueing model, describes how to prepare the information needed for the model, shows the model creation process (with an example), describes each sheet in the clinic planning model workbook, and includes the routing worksheets. The Sample Clinic Model workbook is a completed clinic planning model. It corresponds to the example in the users guide. While it allows the user to change some parameters, it is not possible to change the number of stations (or to add or remove batch processing stations) from any existing clinic planning model.

\section{Spreadsheet Elements}

The software automatically generates a spreadsheet from a minimal set of user inputs. Thus, a planner doesn’t have to understand the mathematics of queueing networks or create a spreadsheet from 
scratch, yet they get a spreadsheet that reflects the design they want to use. The software provides useful data such as the processing time distributions for different types of workstations but allows the user to modify those if necessary.

The planning model workbook consists of five worksheets where the user can enter data and review the results. Each of the worksheets has an assigned color. Blank cells and cells with headers are filled with that color. Cells for entering data and cells that display calculated results have a white background. Navigation buttons along the top of each worksheet are color coded as well. The "Main” worksheet has user inputs in a column on the left and calculated results in a column on the right. The "Parameters" worksheet has some general parameters at the top and a row of parameters for each station below that. The "Routing Table” worksheet has tables for specifying the routing probabilities and distances between stations. The "Staffing” worksheet has a list of blanks for entering position titles and the number of staff in each position. The "Report” worksheet has a less colorful, document-like layout so that the user can print the results. It includes two bar charts of station utilization and queueing.

To simplify the user interface, the clinic planning model doesn't display navigation elements such as sheet tabs and hides the calculation worksheet. However, a user can, without a password, change these options and unhide the calculation worksheet if desired. Thus, a user can see and change any of the spreadsheet details.

The resulting spreadsheets do follow the good spreadsheet design practices (Powell and Baker, 2004). They are modular, with different worksheets for different types of data. A modular design was critical to writing the software that must automatically build the spreadsheet from a template.

The key user inputs (the number of patients to be treated and the number of staff at each station) are located in the same place and are easy to change. The key performance measures are on the same page so that the user can immediately see the impact of changing an input. The calculations are on another, hidden worksheet. Parameters that describe the processing time distribution at each station are clearly shown in a separate worksheet, and the user can change any of them. 


\section{Spreadsheet Dissemination}

To help disseminate the software, we have met with many public health emergency preparedness planners to describe the software and help them get started using it. In February 2006, 32 persons participated in training sessions at a NACCHO conference. These participants had the opportunity to use the software on laptop computers setup in the meeting room, and our team helped the participants build models. In separate workshops, we have helped public health emergency planners from three other counties (two in Maryland, one in Minnesota). The New York State Department of Health used the software to plan 59 POD exercises.

As of May, 2007, our list of registered users has 65 public health emergency preparedness planners from 25 states. Between June, 2005, and May, 2007, the Montgomery County APC received 11 requests for information about the software. In April 2006, 135 persons viewed a web-based seminar about the software.

The Clinic Planning Model Generator is freeware, available for download on our project web site. No payment or registration is required, though we ask users to notify us so that we can add them to our list of users and notify them when updates are available. The University of Maryland retains ownership of the copyright.

Based on the feedback we have received from users, we continue to create updated versions of the software and post them on the web site. We send an email to registered users announcing the release of the new version. However, existing clinic planning models continue to work.

Not charging for the software removes another large obstacle to dissemination and is appropriate because the software is already "paid for" by the sponsor, who wishes to see it distributed to the widest possible audience. Moreover, this approach best fits our institution's vision, which states that the University of Maryland will make "the results of its research available for the use and benefit of the State of Maryland and its people” (University of Maryland, 2000). 


\section{Benefits of Spreadsheets}

The spreadsheet software approach has many benefits. First, due to the popularity of Microsoft Excel, public health emergency preparedness planners do not have to buy new software. The spreadsheet concept and interface are familiar to many, which keeps the time needed to learn the software quite short. The Clinic Planning Model Generator can be used on any computer that has Microsoft Excel. Clinic planning models are portable and self-contained and require only Microsoft Excel. They never become obsolete.

From a development point of view, spreadsheets are suitable for rapid development. Exploiting the existing infrastructure of Microsoft Excel and VBA greatly reduced the software development effort. It also provides a rapid prototyping capability. In discussions with users, we can rapidly modify the clinic planning model to evaluate potential improvements. After identifying a feasible, desirable change, we then modify the Clinic Template and the VBA macros so that all future clinic planning models incorporate that change.

Developing the VBA macros needed to generate clinic planning models from user inputs did require significant effort, but this approach increased the flexibility tremendously. A set of predefined spreadsheet models would have been insufficient.

Unlike discrete event simulation, which requires many long replications to estimate queue lengths and waiting times, a queueing network model implemented as a spreadsheet can calculate performance estimates immediately whenever the user changes a parameter.

We did write and publish a report that presented the mathematical models (Aaby et al., 2006b), but this was not an acceptable alternative to creating a computer-based tool. We do not expect public health professionals to take the time to understand the mathematical models in sufficient detail to implement them. In addition, this would allow many opportunities for mistakes. Table 1 summarizes our qualitative evaluation of the options available. 
Table 1. Evaluation of distribution options for the clinic planning models.

\begin{tabular}{|c|c|c|c|c|c|}
\hline $\begin{array}{l}\text { Distribution } \\
\text { option }\end{array}$ & $\begin{array}{c}\text { Ease of } \\
\text { use }\end{array}$ & Flexibility & Run time & $\begin{array}{c}\text { Need to } \\
\text { buy software }\end{array}$ & $\begin{array}{l}\text { Development } \\
\text { effort }\end{array}$ \\
\hline $\begin{array}{l}\text { Predefined } \\
\text { spreadsheets }\end{array}$ & Very easy & Very low & Very quick & No & Little \\
\hline $\begin{array}{l}\text { Software that } \\
\text { builds } \\
\text { spreadsheets }\end{array}$ & Easy & High & Very quick & No & Moderate \\
\hline $\begin{array}{l}\text { Predefined } \\
\text { simulation } \\
\text { models }\end{array}$ & Fair & Very low & Slow & Yes & Little \\
\hline $\begin{array}{l}\text { Software that } \\
\text { builds } \\
\text { simulation } \\
\text { models }\end{array}$ & Easy & High & Slow & Yes & Moderate \\
\hline New software & Easy & High & Very quick & No & Large \\
\hline $\begin{array}{l}\text { Mathematical } \\
\text { formulas }\end{array}$ & Difficult & High & Very quick & No & None \\
\hline
\end{tabular}

\section{Spreadsheet Development Approaches}

Developing software for distributing spreadsheets to a large set of diverse users required a unique approach that is based on but different from end-user modeling and typical spreadsheet applications.

End-user modeling, a process in which a decision-maker builds and runs his own models, has four key characteristics (Powell, 1997):

1. The decision-maker builds the model.

2. The approach is quantitative but not analytical.

3. The style is quick and dirty.

4. The purpose is to gain insight into a decision or problem.

The three primary steps to building good spreadsheets for end-user modeling are planning, programming, and crafting the user interface (Powell and Baker, 2004). Similarly, Grossman (1999) suggests that end-user modelers first turn off the computer, then write down on paper what the 
spreadsheet will look like, execute the paper spreadsheet by hand, and iterate with previous step, and finally turn on computer and program. An operations research expert can help a decision-maker develop a spreadsheet. Edwards et al. (2000) provide guidelines for this activity and suggestions for good spreadsheet practice.

The other type of spreadsheet development is a spreadsheet application created for a client. Thiriez (2004) provides several examples of professional spreadsheets that he created for others. Based on his experience, he praises the spreadsheet's ease of use, their transparency, the need for no additional software, and the reduction of development effort.

Compared to these processes, the development of the Clinic Planning Model Generator has significant differences. First and foremost, the Clinic Planning Model Generator is not end-user modeling. We were acting as an analyst, constructing models for a client, not as a decision-maker building a spreadsheet for our own use. Because we wanted to provide more flexibility, the typical spreadsheet application approach was insufficient, and we decided that to create a spreadsheet application that, based on user input, automatically modifies a spreadsheet template to generate a custom clinic planning model. Thus, the software approach is similar to source code generation.

From the user’s perspective, the Clinic Planning Model Generator resembles a highly structured enduser modeling process. The user does plan the model, collecting data and writing down on paper the flow of residents through the clinic, using a predetermined form. The user does not have to program the model but merely enters data in a structured way. The user does not have to craft the user interface because it is created automatically. Finally, the user can modify not only the values in the spreadsheet but also the formulas and the source code.

\section{Challenges}

The biggest challenge to this spreadsheet modeling approach has been finding a good compromise between flexibility and structure. We need flexibility because planners want to evaluate a wide variety of 
designs. We need structure to make programming feasible and to reduce the effort needed to learn and use the software.

One of the key reasons for creating a new modeling tool was that existing queueing network analyzers use language that is unfamiliar to public health professionals. We defined queueing terms in language that any lay person can understand and use the correct emergency preparedness terms in our software. Unfortunately, these tend to change over time. For instance, the term "points of dispensing” is now preferred over "mass vaccination clinic.”

From a scientific point of view, the use of approximations means that the spreadsheet provides only estimates of performance. Despite the large body of literature on queueing networks, we had to develop new approximations that are relevant to this domain. We have characterized the accuracy of our estimates and continue working on better approximations (Treadwell and Herrmann, 2005; Pilehvar et al., 2006; Pilehvar, 2007). In this situation, a useful, reasonable approximation is preferable to an exact procedure that is difficult to apply in practice.

\section{Conclusions}

Developing software to create spreadsheets for a large set of diverse users has been part of an exciting process of using operations research to improve planning for bioterrorism attacks, pandemic influenza, or other events. The result is a two-stage process. In the first stage, we develop the software, implemented as macros in a spreadsheet. In the second stage, the user runs those macros to create a customized spreadsheet.

This experience has demonstrated some general lessons for using spreadsheets. From the user's point of view, using spreadsheets eliminates barriers of software purchasing, reduces the amount of training, and provides flexibility because users can easily modify the models to meet their needs. For the application developer, using spreadsheets exploits an existing infrastructure that reduces the development effort and increases the likelihood that users will adopt the application. For the operations research analyst, because the users only enter key values and do not need to understand and program the equations, 
using spreadsheets provides a reliable way to make mathematical models available and reduces the opportunities for mistakes.

The process is significantly different from end user modeling and from developing a typical spreadsheet application for a single client. It has relied upon the ability to conduct rapid prototyping and the cooperation of motivated users.

We are continuing to develop the software based on feedback from the public health professionals using it. Because they are not, fortunately, operating mass dispensing and vaccination clinics, further validation will come from exercises and from similar clinics set up to limit the spread of more common diseases (such as influenza).

\section{Acknowledgements}

The helpful suggestions of anonymous reviewers are greatly appreciated. The spreadsheet software described in this paper is the result of many persons who helped with data collection and modeling, especially Daniel T. Cook, whose time at the University of Maryland was supported by the National Science Foundation under grant EEC 02-43803, and Mark Treadwell, Peter Lin, Ali Pilehvar, and Samuel Fomundam, who developed and enhanced the software in the Computer Integrated Manufacturing Laboratory, a constituent lab of the Institute for Systems Research. Kay Aaby, Rachel Abbey, Carol Jordan, and Kathy Wood at the Montgomery County, Maryland, Public Health Services provided excellent guidance and assistance. Cooperative Agreement Number U50/CCU302718 from the CDC to NACCHO supported this publication. Its contents are solely the responsibility of the University of Maryland and the Advanced Practice Center for Public Health Emergency Preparedness and Response of Montgomery County, Maryland, and do not necessarily represent the official views of CDC or NACCHO.

\section{References}

1. Aaby, K., J.W. Herrmann, C. Jordan, M. Treadwell, K. Wood. 2005. Improving mass vaccination clinic operations. Proceedings of the International Conference on Health Sciences Simulation. January 23-27, New Orleans, Louisiana. 
2. Aaby, K., R. Abbey, J.W. Herrmann, M. Treadwell, C. Jordan, K. Wood. 2006a. Embracing computer modeling to address pandemic influenza in the 21st century. Journal of Public Health Management and Practices 12 (4) 365-372.

3. Aaby, K., J.W. Herrmann, C. Jordan, M. Treadwell, K. Wood. 2006b. Montgomery County’s Public Health Service uses operations research to plan emergency mass-dispensing and vaccination clinics. Interfaces, forthcoming.

4. Centers for Disease Control. 2002. Smallpox response plan and guidelines (Version 3.0). November 26, 2002 http://www.bt.cdc.gov/agent/smallpox/response-plan/

5. Centers for Disease Control. 2006. Pandemic influenza guidance supplement to the 2006 public health emergency preparedness cooperative agreement, Phase II. July 10, 2006.

6. Edwards, J.S., P.N. Finlay, J.M. Wilson. 2000. The role of OR specialists in 'do it yourself' spreadsheet development. European Journal of Operational Research, 127(1) 14-27.

7. Grossman, T.A. 1999. Teachers' forum: spreadsheet modeling and simulation improves understanding of queues. Interfaces, 29(3) 88-103.

8. Kaplan, E.H., D.L. Craft, L.M. Wein. 2002. Emergency response to a smallpox attack: The case for mass vaccination. Proceedings of the National Academy of Sciences 99(16) 10935-10940.

9. National Association of County and City Health Officers. 2005. Advanced Practice Centers. Accessed July 6, 2005 http://www.naccho.org/topics/emergency/apc.cfm.

10. Pilehvar, A., J.W. Herrmann. 2006. Constructing queueing network approximations for mass dispensing and vaccination clinics. Institute for Systems Research, University of Maryland, College Park: Technical Report 2006-17.

11. Pilehvar, A. 2007. Queueing Network Approximations for Mass Dispensing and Vaccination Clinics. M.S. Thesis, Institute for Systems Research, University of Maryland, College Park.

12. Powell, S.G. 1997. The teachers' forum: from intelligent consumer to active modeler, two MBA success stories. Interfaces, 27(3) 88-98.

13. Powell, S.G., K.R. Baker. 2004. The Art of Modeling with Spreadsheets, John Wiley \& Sons, Inc., Hoboken, New Jersey.

14. Thiriez, H. 2004. Spreadsheet-based professional modelling, INFORMS Transactions on Education, 4(2). http://ite.pubs.informs.org/Vol4No2/Thiriez/

15. Treadwell, M., J.W. Herrmann. 2005. Comparing queueing software for mass-dispensing and vaccination clinics. Institute for Systems Research, University of Maryland, College Park: Technical Report 2005-113.

16. University of Maryland, 2000. Building on Excellence: The Next Steps. The Strategic Plan for the University of Maryland, College Park, retrieved July 11, 2006 http://www.provost.umd.edu/Strategic_Planning/Plan.html. 